\title{
Standardizing the Case Narrative
}

\author{
Lisa Rasmussen
}

\section{Introduction}

This chapter is a meta-commentary on case commentaries, in which theoretical questions about method in clinical ethics consultation meet the concrete exigencies of the practice. The difficulty in this task is looking through the lens of a case narrative that is necessarily limited (because it is a distillation of an experience lived through days and weeks of interaction with many people), and a set of commentaries that are themselves limited. The view this perspective may give of a consultation is not the same as the view that might result from an observer accompanying a consultant throughout the case. For example, commenters in this volume uniformly note additional information that was not provided in an already long case narrative. We have no evidence about whether these issues were broached in the case consultation itself; what we do know is that these issues did not appear in the narrative. As a result, this is a book about narrative composition as much as it is about methods in clinical ethics consultation.

But questioning narrative composition of consultation summaries does generate profitable discussion about the field of clinical ethics consultation itself. As we move towards standardization and quality improvement in clinical ethics consultation, peer review is a vital component for evaluating consultants and consultations. In this, we are hampered by the fact that there is no sustainable mechanism for allowing peer review of full consultations, and even less chance of observing a single consultant's practice over many cases. Instead, we have the proxy (as currently utilized in the Attestation model [Kodish et al. 2013]) of the consultant's case study. A flaw in this model is the possibility that excellent consultants can be poor writers, and that gifted writers may be poor consultants (where by "gifted" and "poor"

\author{
L. Rasmussen $(\bowtie)$ \\ University of North Carolina - Charlotte, Charlotte, NC, USA \\ e-mail: 1rasmuss@uncc.edu
}


writing I am referring to narrative compositional choices in addition to word or style choices). If we are to be evaluated by our case narratives, we must try to control for features irrelevant to quality evaluation, such as narrative ability, rhetorical skill and seductive rococo vocabulary. ${ }^{1}$

We may also want to control for the consultant's perspective: What we choose to accent in a narrative is what is important or noteworthy about the case to $u s .^{2}$ We may not be lying or adorning a story, but we are choosing a perspective from which to view a case, and when those choices are made in composing the case study, other possible choices become invisible. There are standard elements that may be almost universally expected in case summaries (such as identifying whether a patient is competent or has decisional capacity, and if not, who the decision maker is and under what governing rule [legal default, HCPA, etc.]), but many aspects of what is noteworthy about a case will be idiosyncratically determined. A person sensitized to issues of faith, gender, culture, or socioeconomic status may focus on or preferentially include these issues, where another person might personally note the presence of such features during the case consultation itself but not center or even mention them in a particular case narrative if they do not play a significant role.

The conclusion I have arrived at after considering "The Zadeh Scenario" and accompanying peer reviews is that if quality attestation is to be largely evaluated based on the case narratives submitted to the judging panel, we must develop a fairly uniform standard for case reports themselves, which is distinct from developing a uniform standard for how a consultation is conducted. ${ }^{3}$ In what follows, I note several features of the peer reviews accompanying the Finder case that belie different

${ }^{1}$ For example, consider some of the ways commenters describe Finder's narrative: "wondrously rich;" "polished;" "wonderfully detailed and engaging;" "rich and thoughtful;" a "gift." Though some of this praise is (rightfully) due to gratitude for the fact that Finder makes himself vulnerable in this new mechanism for peer review, it is also an honest assessment of the way in which the narrative is written. Clearly the commenters did not shirk from challenging elements of the narrative due to their praise, but we know from the fields of psychology and sociology that style affects assessment. For example, in the "illusory effect," a message delivered repeatedly is believed more frequently. By repeating claims about "caring for" or "listening to" a patient or family, a consultant could affect an assessment of the case narrative (and thus, the consultation itself) as including evidence that a consultant had the right attitude towards the participants.

${ }^{2}$ As Tod Chambers puts it in his volume about the topic of written cases, The Fiction of Bioethics, "What ethicists have generally ignored is that cases - the data by which they test the relevance of moral theory - are fictions. That is, they are made up, constructed and thus follow conventions of representation that inevitably bias how one understands this information" (1999, p. 10; emphasis added).

${ }^{3}$ Obviously in this case, Finder and Bliton have not conceived of the volume as focusing on what they argue is a paradigm case narrative. The purpose of the case narrative for the volume and the purpose of case studies written as part of a dossier for quality evaluation may be quite different. Therefore my comments are not directed at this volume's device in particular, but rather, at the more general activity of evaluating consultants and consultations via written reports. (This also raises the question of what to call such writing: "case study" implies a quite distilled summary of a case, while "case narrative" implies a more contextualized account that invites the reader to consider stakeholders more as characters, with personalities, interior lives, and motivations. A discussion of what we should expect from cases summarized for attestation purposes should start with what to call them, and what general tenor they should have within this continuum of possibilities.) 
notions of what should be included in a case summary. This may -- or may not -- in turn belie different notions of what questions should be explored as part of doing clinical ethics consultation as a practice.

\section{Disparate Targets of Commentaries}

The overall perception a reader of the case and peer reviews is left with is of an impressionistic landscape. Reviewers pick and choose, some working chronologically through the case, others simply picking up pieces they find noteworthy and elaborating on their presence or absence from the case narrative. The organization and focus of each peer review is sui generis; they are anything but standardized. While there are a few commonalities, most notably with respect to procedure, the peer reviews diverge quite significantly from each other. Two articles observe that it is not immediately obvious that this is a clinical ethics consultation (Tarzian, who concludes after discussion that it is a case consultation, and Rosell \& Johnson, who ask but do not explicitly answer this question).

I think this unsystematic approach accurately represents the state of the field. But this must be changed if quality improvement is really what we are after. Systems help to control for the fact of cognitive limitation. We all know healthcare providers should wash their hands, for example, but it took a fairly rigid study and assurance mechanisms to reveal how frequently that did not happen and how controlling for hand-washing and other straightforward, already-verified practices could dramatically affect patient well-being (Haynes et al. 2009). Establishing a system that controls for human cognitive limitations (such as forgetfulness) merely does a better job of making what is desired actually happen than does leaving it up to chance. If case narratives are to be the coin of the new realm of attestation and quality improvement, systematicity in reporting is necessary. ${ }^{4}$ Systems establish standards to which individual instances can be compared for adherence; without a system in case reporting, for example, we cannot immediately judge whether a consultation was poor, middling, or excellent based on how the report is written or on what is and is not included. If all we have to evaluate the case is a written report, and we lack a standard for evaluating the written report, we lack a true standard for evaluating a case. And so, questions about method in consultation become, under an attestation model, questions about method in consultation summaries. We must articulate the links between what should be done in a consultation and what must be included in a case report.

To illustrate this point, consider two categories of observations represented in Part Two's peer reviews: proceduralism, and feminist and multicultural issues. Not

\footnotetext{
${ }^{4}$ I recognize that a likely consequence of systematizing or standardizing the case report will be a flattening of richness and detail. But flattening of variation is exactly the point of systems and standards, and it is not clear to me how one might steer between this Scylla and Charybdis. However, it does suggest that case studies or reports for credentialing purposes (in which careers might eventually be at stake) can exist simultaneously with richer narratives meant for other venues.
} 
all peer reviewers mention both; procedural concerns are cited in four of the five peer reviews, cultural concerns by only three, and feminist concerns by only one. Several other concerns are each mentioned by only one peer reviewer. What do these differences tell us about the practice of consultation and about the practice of writing consultation reports?

\section{Proceduralism}

By "proceduralism," I mean a focus on proper consultation procedures as evidenced by the case narrative. Tarzian focuses on proceduralism most clearly, as evidenced in her subtitle, "A Focus on Process," and notes that her experience as a chair and member of two different task forces on setting standards in clinical ethics consultation left her with "an appreciation for procedural standards in health care ethics consultation" (Tarzian 2018: 75). Her initial comments, for example, focus on whether this case actually constituted an ethics consultation, and whether it was a case consultation or something else; to answer these questions, she turns to the Core Competencies for Healthcare Ethics Consultation (ASBH 2011; hereafter "CC"), the current standard in the field, if there is one. Later, she observes that in "The Zadeh Scenario," "there is ambiguity in several procedural aspects of how this consultation was handled," such as whether the role of the former consultant (Moore) was clarified for staff and whether Finder clarified what role he himself would play in the case, with the ultimate question being, Who is really in charge of the case and of communicating with various stakeholders? (Tarzian 2018: 79). Another ambiguity Tarzian comments on is the extent to which medical uncertainty (and the accompanying ethical uncertainty) regarding appropriate treatments was articulated both for health care providers and the patient's family; she points out that "a strategy for determining how these decisions are made" is missing (Tarzian 2018: 83).

Armstrong similarly focuses on procedures by commenting positively on the fact that Finder prepares for the consultation by reviewing the electronic medical record and notifying the attending that he is now involved in the case. However, like Tarzian, Armstrong also wonders whether Finder clarified his role with the family, and why an account of who is making decisions (and why) is missing from the narrative. ${ }^{5}$ As practicing CECs will know, there are probably more "informal" or "curbside" consultations than formal ones, and Armstrong points out that "a line can and should be drawn between creating a safe space to discuss and examine moral feelings and a responsibility to follow-up and take action on issues uncovered during such discussions." Because Finder describes both formal and informal requests for consultation, yielding uncertainty about which this case should involve, Armstrong is highlighting the need for the field to create appropriate procedures for these different kinds of consultations.

\footnotetext{
${ }^{5}$ Armstrong goes further than Tarzian with this concern, offering an extended commentary on "the missing patient" in Finder's narrative.
} 
Hynds' first comment is that consultations should occur "upon request" (that is, not "self-authorize[d]"), so as to avoid the role of the Ethics Police, and observes that it is not clear whether the first consultant in this case (Dr. Moore) was formally consulted. He also wonders about the presumably "hierarchic relationship" between Dr. Finder and Dr. Moore (Hynds 2018: 92) and about the "seemingly unstructured or semi-structured" engagement of consultants with participants (Hynds 2018: 89). He recommends, too, that while Finder explains that consultants in this case met with stakeholders multiple times (and alone), it is better if "interventions are kept to a minimum and all the main players are generally present" (Hynds 2018: 90). The main procedural concerns in Hynds' peer review thus concern the formality and source of the consult request, the appropriate structure of interactions with stakeholders, and the professional relationship between consultants.

Frolic \& Rubin, like Tarzian and Armstrong, comment on the apparent lack of role clarity in this case. Not only was the role unclear, they suggest, other procedures were as well. For example, procedural clarity was not evident in this case regarding the "various phases of ethics consultation;" it is not clear from the narrative whether the "ethics consultation in this case contributed to any positive outcome, beyond a good relationship between Finder and the family" (Frolic and Rubin 2018: 59); and it is unclear "if and how the voices of the bedside staff were included in the consultation process" (Frolic and Rubin 2018: 59). Many of these procedural issues are attributed to a lack of "formalization of both the ethics consultant's role and process" (Frolic and Rubin 2018: 59).

Four of the five peer reviewers included procedural concerns in their remarks, ${ }^{6}$ but it is noteworthy that they did not all cite the same procedural concerns. The most frequently cited procedural issue was that "The Zadeh Scenario" does not demonstrate that Finder made his role, or that of his colleague Dr. Moore, clear to the patient's family or the medical staff. (It is worth noting, though, that he may have and simply chose not to mention this in the narrative.) Beyond this node of agreement, however, stated concerns about procedure vary. Armstrong mentions the importance of distinguishing between formal and informal consultations; Hynds stresses the importance that consultants should wait to be called (rather than being proactive) and that consultation meetings should usually involve all stakeholders; and Frolic \& Rubin observe that a lack of formalization of the process of consultation may be causing a number of problems.

The peer reviews are not mutually incompatible, so I do not mean to suggest that they demonstrate some radical disagreement about clinical ethics consultation. But they do present quite different pictures of what a case narrative should include. This

\footnotetext{
${ }^{6}$ I have not included in this count Rosell \& Johnson, who describe their commentary as a phenomenological analysis in an "interrogative" mode and do not couch their comments in "procedural" terms. However, many of their remarks may have procedural elements - for example, they note that patients have the right not to receive particular treatments or care from particular providers, and that they have the right under law and because of respect for autonomy to make decisions for themselves.
} 
may help explain the focus on procedure, because it is an area where we might hope for some consensus. However, even in the comments on procedural issues, these peer reviews reveal different priorities.

\section{Multicultural and Feminist Perspectives}

A quote from Dr. Moore indicates that the family is Persian, and three of the peer reviewers take up this fact for discussion. Rosell \& Johnson observe only in passing that "the narrative gives several indicators of cultural normative difference in regard to making end of life decisions" (Rosell and Johnson 2018: 103), while Tarzian and Armstrong offer more extended comments. Tarzian raises the idea of "cultural competence" in providing health care, and asserts that "[i]t's clear that this [cultural difference] contributed substantially to the perceived conflicts between the Hamadani family and the staff caring for Mrs. Hamadani” (Tarzian 2018: 78). However, she notes that although Finder recognized these possible cultural issues in the case, "it's unclear whether or how he addressed these conflicts in his role as the ethics consultant" (Tarzian 2018: 80). Armstrong challenges whether we should assume, based on the family's Persian culture, that the patient would have wanted her family to make decisions for her.

Encountering a patient and family with a cultural foundation that might affect their approach to decision-making is common in clinical ethics consultation. Cultural difference can be a linchpin for an entire case - or completely irrelevant. Here, two out of five peer reviews did not even include mention of the issue; but even of the three that did, the responses to the cultural element of the case have next to no overlap. To be specific, one flags it but does not assess Dr. Finder's performance or narrative based on it; one assumes that the cultural issue "contributed substantially to the perceived conflicts" and faults Finder for not discussing how he incorporated these concerns into his consultation; and one notes that culture is not dispositive of wishes when a patient has not stated her own wishes.

Gender and social norms can also play a significant role in clinical ethics cases, but only one author, Armstrong, comments on the role of gender in the case at hand. This case involves an incapacitated mother with one son and two daughters, but the only apparent candidate for decision-maker appears to be the son. Armstrong observes that the daughters have only first names but the son has a first and last name (and is called "Mr. Zadeh" in the narrative), and that "none of the female characters' opinions regarding treatment independent of Mr. Zadeh's interpretation are explored...both the patient and her daughters form a silent chorus for Mr. Zadeh" (Armstrong 2018: 69). It is worth noting that only one of the five peer reviewers found the issue of gender worth noting. 


\section{Other Issues}

In addition to procedural and cultural concerns, several other concerns are mentioned in Part Two, though in most cases only one author mentions each feature. Armstrong, Rosell \& Johnson, and Frolic \& Rubin all focus on the absence of the patient's voice in "The Zadeh Scenario," but mostly in passing. Rosell \& Johnson remark that "Mrs. Hamadani is mostly absent in this consultation activity" (Rosell and Johnson 2018: 106); Frolic \& Rubin raise the question about what the patient wants as the final and most important category in a list of questions that are not answered in the narrative; Armstrong, in her discussion of "the missing patient," notes that by not mentioning any conversation with the patient about her wishes, "the case appears to presume that she did not wish to be involved in decision-making, or that her wishes were adequately represented by her children" (Armstrong 2018: 68).

Armstrong and Frolic \& Rubin comment on Finder's use of the electronic medical record to track readmitted patients who have had ethical issues during past admissions. Frolic \& Rubin "worry this could lead to some inadvertent role confusion (what exactly is the ethics consultant contributing by 'checking in' on a previous case?) as well as potential violation of the patient's privacy" (Frolic and Rubin 2018: 58). Armstrong also mentions privacy, but emphasizes more that this practice makes it difficult to know when a consultation has ended, and whether follow-up in this way is part of continuity of care.

Frolic \& Rubin also stress the importance of a consultant's self-examination and reflecting critically on one's own practices, noting that Finder does not engage in this practice in this narrative. Armstrong comments that there was evidence of moral distress among healthcare providers, but that Finder does not indicate that this issue was explored at all in the narrative.

\section{Reflections}

The peer reviews in Part Two, like "The Zadeh Scenario," are rich, thoughtful, and appropriate. But even if all of them are correct, I cannot help but conclude that no case narrative short of a novella could hope to satisfy all of these informational demands, and perhaps not even then. This is not a judgment about the authors of the case or peer reviews, who are attuned to the limits of this format. They also did not compose their comments on the premise that they were working towards some paradigm in case narrative. This book is not meant to recommend a model for adoption in case studies. However, because Finder's case is (as the peer reviewers note) a much richer, more contextual and detailed narrative than is customary, and yet still all five authors in Part Two find much lacking in the description, the conclusions drawn must also hold for case studies in general given that they tend to be much 
shorter, more generic, and lacking in detail and context. The case study is a limited vehicle, in other words, and if it is to form the basis of attestation in clinical ethics consultation, we must standardize what we expect to see in a case narrative.

Imagine if the peer reviewers on this case comprised an attestation panel. Although there is some overlap in their positive comments (e.g., most praised Finder for the care he showed the family), there is hardly any overlap in their critical comments. What would this mean for an attestation evaluation of Finder? This returns us to the difference between assessing a consultant's practice and assessing a case report, because the assumption in the attestation model is that the practice can be evaluated via the case report. This assumption is problematic.

First, if these peer reviews are any indication, authors will have idiosyncratic framing mechanisms for writing case reports, and evaluators will have idiosyncratic responses to cases. For example, Finder may have noticed, explored, and eliminated a feminist concern from the narrative because it played no role, and only one commenter (Armstrong) mentions feminist concerns in her paper. The lack of mention of feminist concerns in the case is merely absence of evidence, not evidence of absence. Armstrong's concern with the way the case narrative is written (e.g., that the daughters have only first names and the son a first and last name) may be legitimate, but it may be a concern with Finder's narrative choices, not a concern with his consultation practice (except by tenuous inference between narrative choices and consultation practices).

Three peer reviewers mention cultural issues, reacting to Dr. Moore's statement that the family is Persian and to Finder's conclusion that "cultural elements" may have played a role in the case. But there are many more possible interpretations of this case narrative. We have no actual evidence that culture was at play, rather than religion, family dynamics, economic issues, etc. The fact that the family is Persian (are the children also Persian? Or are they Persians born in the US?) ends up being an easy heuristic for "value difference," but it is a heuristic that may mislead. It is not implausible that culture affected the case, but culture is not monolithic, and we characterize individuals inappropriately if we assume that individual beliefs follow directly from cultural context. Finder may have been blind to these issues, exquisitely attuned to them, or somewhere in between; the commenters may have chosen to focus on "pet" concerns, may have drilled right to the heart of the case, or somewhere in between. We simply lack the information we would need to establish which is the case.

In the account of the proposed Attestation model (Kodish et al. 2013), the authors recognize the problem of interrater reliability (which is part, but not all, of the problem outlined above) and offer a mechanism to address it. As the evaluation of the portfolios moved forward, the 12 members of the assessment group "convened to establish assessment metrics" based on the portfolios they had received, then piloted the instruments for face validity and construct validity. It is the problem of "construct validity" - "the ability of the test to measure what is intended" - that I mean to focus on. What I have been illustrating in discussing these case commentaries is the problem of our lack of standards in case reports. If we think it is important to include in a case report a discussion of even those issues that played no significant role during the case, we must offer a template or framework of case reports that 
prompts discussion of such issues, even if that amounts to a statement that this issue was not relevant to the case. ${ }^{7}$

\section{Conclusion}

Quality improvement procedures only work if they capture all and only what is appropriate to the task. We have a double-layer problem in the idea of an attestation model of assessing clinical ethics consultation, because we lack standards at the level of the individual consultation, and we haven't even begun to discuss standard expectations of case summaries. This model of an extended case study with commentaries is worth repeating as we develop standards in the field, because such a process generates the diverse elements of a consultation and its reporting that practitioners find important. ${ }^{8}$ As the field's assessment mechanisms are shaped, these elements must be standardized and promulgated so that authors of case reports know the bases on which their cases will be assessed. Without clear direction regarding the necessary elements in a case report, attestation of abilities based on case summaries will be impossible, unfair, or arbitrary.

\section{References}

Armstrong K (2018) Telling about engagement is not enough: seeking the "ethics" of ethics consultation in clinical ethics case reports. In: Finder SG, Bliton MJ (eds) Peer review, peer education, and modeling in the practice of clinical ethics consultation: the Zadeh project. Springer, Cham, pp 63-73

Chambers T (1999) The fiction of bioethics: cases as literary texts. Routledge, New York

Finder SG (2018) The Zadeh scenario. In: Finder SG, Bliton MJ (eds) Peer review, peer education, and modeling in the practice of clinical ethics consultation: the Zadeh project. Springer, Cham, pp 21-42

Frolic A, Rubin SB (2018) Critical self-reflection as moral practice: a collaborative meditation on peer review in ethics consultation. In: Finder SG, Bliton MJ (eds) Peer review, peer education, and modeling in the practice of clinical ethics consultation: the Zadeh project. Springer, Cham, pp 47-61

Haynes AB, Weiser TG, Berry WR, for the safe surgery saves lives study group et al (2009) A surgical safety checklist to reduce morbidity and mortality in global population. NEJM 360:491-449

\footnotetext{
${ }^{7}$ One possibility for ensuring comprehensiveness both during a consultation and in a case report is the use of a standardized checklist. I gave a presentation on such a mechanism at the International Conference on Clinical Ethics Consultation 2015, in New York, NY.

${ }^{8}$ For example, journals could add a regular feature emulating this approach, and conferences such as the American Society for Bioethics and Humanities (ASBH) and the International Conference for Clinical Ethics consultation could provide conference slots for panels arranged for this commentary.
} 
Hynds J (2018) Ethics consultation, professional praxis, and what it means to be a "consultant". In: Finder SG, Bliton MJ (eds) Peer review, peer education, and modeling in the practice of clinical ethics consultation: the Zadeh project. Springer, Cham, pp 85-97

Kodish E, Fins JJ, Braddock C et al (2013) Quality attestation for clinical ethics consultants: a two-step model from the American Society for Bioethics and Humanities. Hastings Cent Rep 43(5):26-36

Rosell T, Johnson B (2018) This may, or may not, be an ethics consultation. In: Finder SG, Bliton MJ (eds) Peer review, peer education, and modeling in the practice of clinical ethics consultation: the Zadeh project. Springer, Cham, pp 99-107

Tarzian AJ (2018) Ethics consultation for Mrs. Hamadani: a focus on process. In: Finder SG, Bliton MJ (eds) Peer review, peer education, and modeling in the practice of clinical ethics consultation: the Zadeh project. Springer, Cham, pp 75-84

Open Access This chapter is licensed under the terms of the Creative Commons Attribution 4.0 International License (http://creativecommons.org/licenses/by/4.0/), which permits use, sharing, adaptation, distribution and reproduction in any medium or format, as long as you give appropriate credit to the original author(s) and the source, provide a link to the Creative Commons license and indicate if changes were made.

The images or other third party material in this chapter are included in the chapter's Creative Commons license, unless indicated otherwise in a credit line to the material. If material is not included in the chapter's Creative Commons license and your intended use is not permitted by statutory regulation or exceeds the permitted use, you will need to obtain permission directly from the copyright holder.

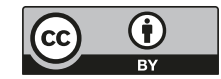

